\title{
Impulse Radio Ultra Wide Band Based Mobile Adhoc Network Routing Performance Analysis
}

\author{
${ }^{1}$ Sreedhar, M. and ${ }^{2} \mathrm{C}$. Venkatesh \\ ${ }^{1}$ Department of Electrical and Electronic Engineering, \\ Velalar College of Engineering and Technology, Erode, India \\ ${ }^{2}$ Department of Electronic and Communication Engineering, \\ EBET Group of Institutions, Nathakadiyur, India
}

Received 2012-05-15, Revised 2012-09-02; Accepted 2013-05-02

\begin{abstract}
This study presents with the routing overhead analysis of impulse radio - Ultra Wide Band based wireless networks which Employs Dynamic Nature Routing (EDNR) method for determining and selecting the stable route in Mobile Adhoc Network (MANET). This method requires only two sample packets and uses piggyback information on Route-Request (RREQ) and Route-Reply (RREP) packets during a route-discovery procedure. Conventional ENDR results in better routing overhead and packet delivery ratio characteristics with higher energy consumption. The proposed method explores the characteristics of UWB nature of routing based on Node lifetime and Link lifetime prediction for reducing the required bandwidth while maintaining higher packet delivery and lower routing overhead. Simulation results illustrate the improvement of packet delivery ratio by $5 \%$ and reduction of routing overhead by $4.8 \%$ of the proposed UWB EDNR method over the conventional EDNR method. For higher packet delivery ratio and reduced routing overhead with efficient bandwidth utilization can be achieved by applying UWB based EDNR routing that is desired for real time data communications.
\end{abstract}

Key words: UWB, QoS, MANET, Routing, Employs Dynamic Nature Routing (EDNR), Method Requires, Routing Overhead, Bandwidth Utilization, Piggyback Info, Link Lifetime

\section{INTRODUCTION}

A Mobile Ad hoc Network (MANET) represents a system of wireless mobile nodes that can self-organize freely and dynamically into arbitrary and temporary network topology. A mobile ad-hoc network (MANET) is a self-configuring infrastructureless network of mobile devices connected by wireless links.

\subsection{Routing Overhead}

Sending a payload of data (reliably) over a communications network requires sending more than just the desired payload data, itself. It involves sending various controls and signaling data (TCP) required for achieving the reliable transmission of the desired data. The control signaling used for communication is called overhead.

\subsection{Packet Delivery}

The packet delivery ratio is defined as the number of data packet received by destination nodes divided by number of data packets transmitted by source nodes.

\subsection{Routing Protocols in MANET}

An ad-hoc routing protocol is a convention that controls how nodes decide which way to route packets between computing devices in a mobile ad hoc network.

In ad-hoc networks, nodes are not familiar with the topology of their networks; instead, they have to discover it. The basic idea is that a new node may announce its presence and should listen for announcements broadcast by its neighbors. Each node Corresponding Author: Sreedhar, M., Department of Electrical and Electronic Engineering, Velalar College of Engineering and Technology, Erode, India 
learns about nodes nearby and how to reach them and may announce that it, too, can reach them.

Different protocols are evaluated based on the packet drop rate, the overhead introduced by the routing protocol, end-to-end packet delays, network throughput.

Existing routing protocols are having the drawbacks as listed:

- Nodes speed is moderate

- Route breaking

- High energy consumption

- Flooding

- High end to end delay

The above drawbacks can be overcome by applying EDNR algorithm for routing in MANET as proposed by Zhang et al. (2010) and Bettstetter et al. (2003).

\subsection{Exploring Dynamic Nature Routing (EDNR)}

This algorithm consists of the following three phases: route discovery, data forwarding and route maintenance.

Every node saves the received signal strength and the received time of the RREQ packet in its local memory and adds this information into the RREP packet header in a piggyback manner when it receives the RREP for the corresponding RREQ packet to meet the requirement of the connection lifetime-prediction algorithm.

Second, node agents need to update their predicted node lifetime during every period. Finally, the nodelifetime information in the RREP packet is updated when the RREP packet is returned from a destination node to the source node.

At every EDNR node agent, a variable NLT, which represents the node lifetime, is added to represent the estimated lifetime of this node and it is updated by the algorithm.

For the lifetime of a link $\mathrm{Ci}$, there are two sample packets exchanged between nodes $\mathrm{N}_{\mathrm{i}}-1$ and $\mathrm{Ni}$ (packet 1: $\mathrm{N}_{\mathrm{i}}-1$ RREQ $\rightarrow \mathrm{N}_{\mathrm{i}}$; packet 2: $\mathrm{N}_{\mathrm{i}}-1$ RREP $\leftarrow \mathrm{N}_{\mathrm{i}}$ ) in the route-discovery phase and thus, the LLT using the proposed algorithm can be estimated.

Node $\mathrm{N}_{\mathrm{i}}$ knows the RREP time and the RREP received signal strength from node $\mathrm{N}_{\mathrm{i}}+1$ (information of $\mathrm{N}_{\mathrm{i}}$ RREP $\leftarrow \mathrm{N}_{\mathrm{i}}+1$ ). Thus, it can obtain the second sample packet that is delivered between the corresponding two nodes $\left(\mathrm{N}_{\mathrm{i}}, \mathrm{N}_{\mathrm{i}}+1\right)$ and thus, the connection time TCi can be calculated using the connection lifetime-prediction algorithm and then update the local LLT value.

Similarly, node $\mathrm{N}_{\mathrm{i}}$ should add the RREQ Info entry that is received from nodeN $\mathrm{i}^{-1}$ to the RREP header before sending the RREP to node $\mathrm{N}_{\mathrm{i}}-1$ and then node $\mathrm{N}_{\mathrm{i}}$ 1 calculates the LLT between two nodes.
Three new entries, i.e., Path Lifetime (PLT), RREQ time and RREQ signal strength, are added to the common header of an RREP packet.

The PLT represents the predicted lifetime of the source route in this packet header and can be updated when RREP packets are forwarded from the destination node to the source node in the route-discovery phase.

The RREQ time and the RREQ signal strength represent the RREQ Info of the previous RREQ node. The EDNR node agent only updates the PLT value in the common header of the RREP packet with a local NLT value or LLT value, if NLT $<$ PLT or LLT $<$ PLT, before forwarding this RREP packet.

When this RREP packet reaches the source node, the PLT becomes the minimum value of the estimated lifetime of all nodes and links through the route from the source node to the destination node.

In the persistent data forwarding period, a source node tends to select the path with the longest lifetime from multiple paths as a source route for data forwarding.

\subsection{Node Lifetime Prediction Algorithm}

Two nodes that have the same residual energy level, an active node that is used in many data-forwarding paths consumes energy more quickly and thus, it has a shorter lifetime than the remaining inactive node.

The node lifetime that is based on its current residual energy and its past activity solution that does not need to calculate the predicted node lifetime from each data packet as proposed by Bettstetter et al. (2003).

Used an exponentially weighted moving average method to estimate the energy drain rate $\mathrm{ev}_{\mathrm{i}}$. $\mathrm{E}_{\mathrm{i}}$ represents the current residual energy of node $i$ and $e v_{i}$ is the rate of energy depletion.

The estimated energy drain rate in the $n^{\text {th }}$ period is $\mathrm{ev}_{\mathrm{i}}{ }^{\mathrm{n}}$ and $\mathrm{ev}_{\mathrm{i}}{ }^{(\mathrm{n}-1)}$ is the estimated energy drain rate in the previous $(n-1)^{\text {th }}$ period, $\alpha$ denotes the coefficient that reflects the relation between $e_{i}{ }^{n}$ and $e^{n}{ }^{n-1}$ and it is constant value with a range of $[0,1]$.

\subsection{Link Lifetime-Prediction Algorithm}

As proposed by Gerharz et al. (2002) and Maleki et al. (2003), by assuming that senders transmit packets with the same power level, a receiver can measure the received signal power strength when receiving a packet and then calculates the distance by directly applying the radio propagation model. If the received signal power strength is lower than a threshold value, this link is considered as an unstable state and then calculates the connection time. The relative motion of two nodes $\left(\mathrm{N}_{\mathrm{i}}-1, \mathrm{~N}_{\mathrm{i}}\right) \mathrm{t}$ relative velocities $\mathrm{v}_{\mathrm{i}}$ and $\left(\mathrm{v}_{\mathrm{i}}-1\right)$ relative to ground at a given time $\mathrm{t}$. 


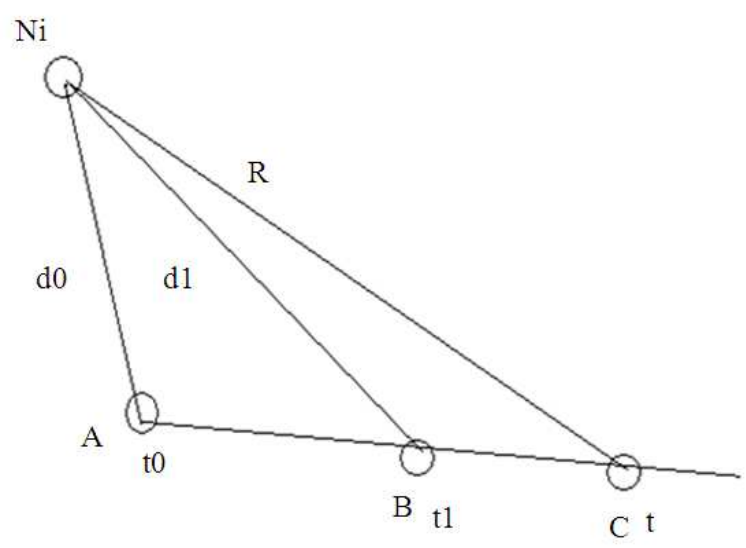

Fig. 1. LLT prediction algorithm

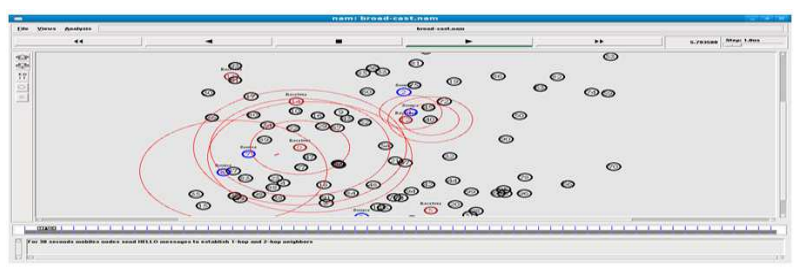

Fig. 2. Establishing one hop and two hop neighbors

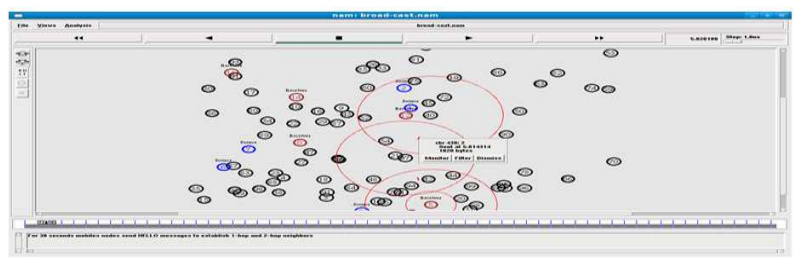

Fig. 3. Traffic creation

Table 1. Simulation parameters

\begin{tabular}{ll}
\hline Simulation time & $100 \mathrm{~s}$ \\
\hline Number of nodes & 100 \\
MAC type & MAC 802.11 \\
Model and range & Two ray model, 250 \\
$\mathrm{m}$ & \\
Max speed & $4-24 \mathrm{~m} \mathrm{sec}^{-1}$ \\
Initial energy & $100 \mathrm{~J}$ \\
Traffic and rate & CBR $512 \mathrm{bps}$ \\
No. of connections & 50 \\
\hline
\end{tabular}

The ground is used as a reference frame by default. If node $\mathrm{Ni}$ is considered as the reference frame, node $\mathrm{N}_{\mathrm{i}}$ 1 is moving at relative velocity of $\mathrm{v}$, as given by the following: $\mathrm{v}=\mathrm{v}_{\mathrm{i}-1}-\mathrm{v}_{\mathrm{i}}$. To calculate the connection time $\mathrm{TC}_{\mathrm{i}}$, apply a triangle geometry as shown in the Fig. $\mathbf{1}$.
This predicts the link expiration time for reactive route maintenance in the previous work.

Proposed method requires only sample packets and implements piggyback information on Route-Request (RREQ) and Route-Reply (RREP) packets during adiscovery procedure with no other control message overhead and thus, it does not increase time complexity.

\subsection{Establishing One Hop and Two Hop Neighbors}

Figure 2 shows the simulation environment in which 100 nodes are placed. The environment is created as per the characteristic of MANET. The nodes are thus developed and they are designed as randomly movable. During data transmission the nodes will be acting as router.

\subsection{Traffic Creation}

The Fig. 3 describes the transmission between sender and receiver. EDNR is used to find out the shortest path distance between the two nodes while transmission. Each host is equipped with a radio transceiver. The Two Ray Ground model is used to predict the signal power received by the receiver. CBR data traffic flows are injected into the network from the servers and the size of the data payload is 512 bytes. In two ray ground model, to transmit the signal a single line-of-sight path between two mobile nodes is the only means of propagation. The MAC protocol uses a CSMA/CA.

\subsection{Simulation Parameters}

The network environment has been created and simulation is carried out in NS2 for analyzing the route lifetime algorithm. The following Table 1 shows the important parameters chosen for the NS2 simulation.

\subsection{EDNR Algorithm Inference}

The graphical analysis shows the performance of the EDNR algorithm. The Fig. 4 shows the characteristics of number of packets received at different receiver sinks.

The Fig. 5 shows the packet delivery ratio characteristics of the simulated network. This method results in reduced number of packets at sinks and packet delivery ratio has been considerably reduced.

In order to further improving the packet delivery ratio and reducing the routing overhead with efficient utilization of available bandwidth, the new scheme has been proposed based on impulse carrier routing i.e. UWB communication which employs Node Life Time and Network Life Time Prediction algorithms. 


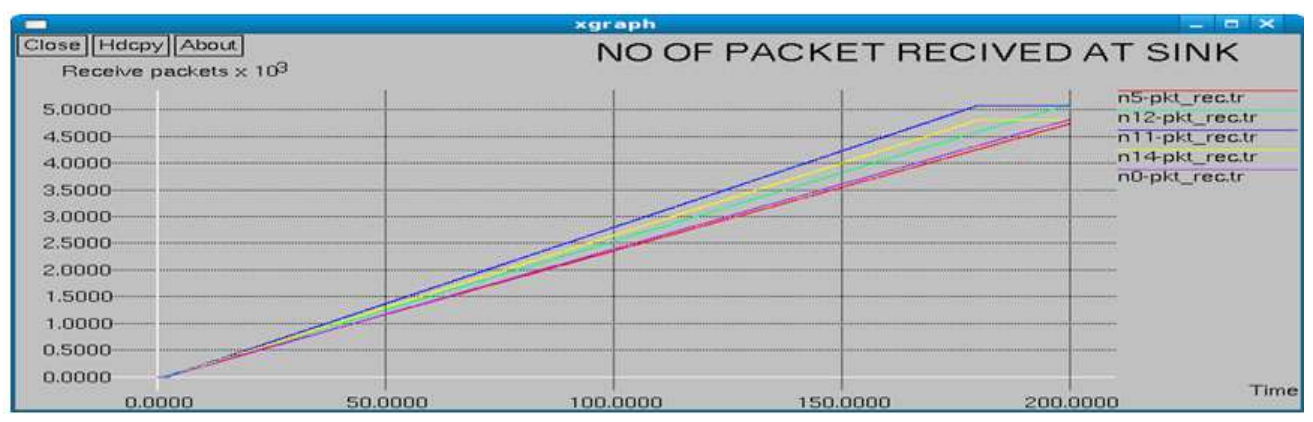

Fig. 4. Number of received packets at sink

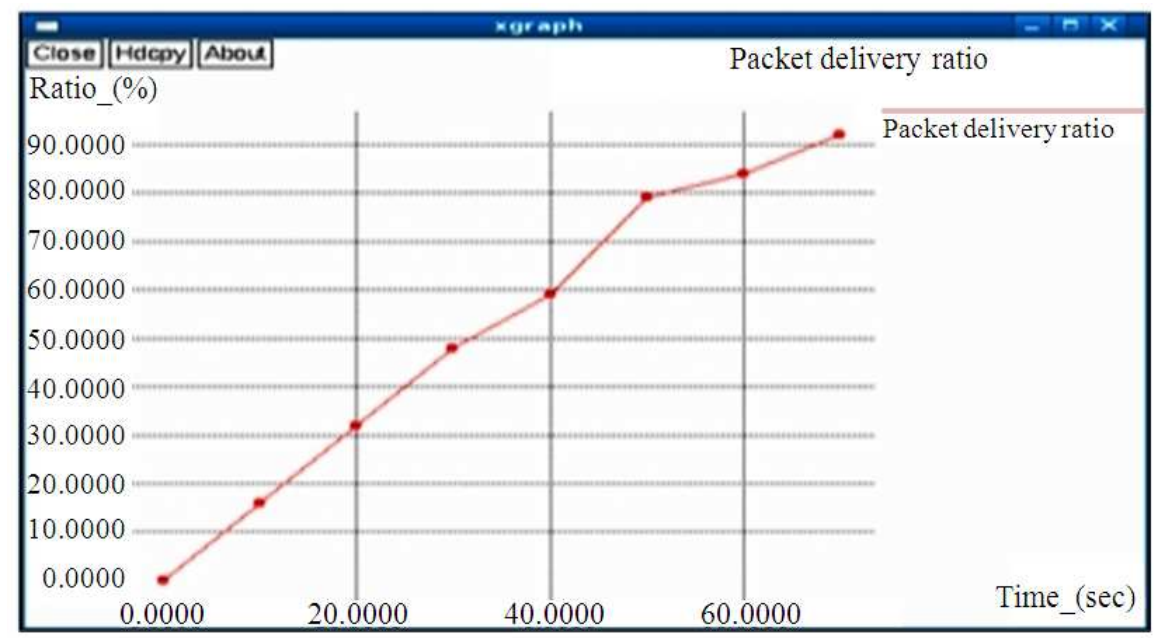

Fig. 5. Packet delivery ratio

\section{MATERIALS AND METHODS}

The proposed system uses UWB pulses for transmitting the data packets by exploring Dynamic Nature Routing method for determining and selecting the stable route.

It uses the Node lifetime prediction method and Link lifetime prediction methods for selecting the stable route with long lifetime.

\subsection{Ultra Wideband}

Ultra-Wideband (UWB) is a radio transmission scheme that uses extremely low power pulses of radio energy spread across a wide spectrum of frequencies.

UWB has several advantages over conventional continuous wave radio communications including potential support for high data rates, robustness to multipath interference and fading.
Table 2. UWB characteristics

\begin{tabular}{ll}
\hline Fractional bandwidth & $>20 \%$ \\
\hline Pulse width & 0.1 to $0.2 \mathrm{~ns}$ \\
Pulse repetition frequency & $1 \mathrm{Khz}$ to $2 \mathrm{Ghz}$ \\
Average transmitted power & $<1 \mathrm{~mW}$ \\
\hline
\end{tabular}

\subsection{UWB Based Routing}

Ultra-Wideband also known as baseband or impulse radio is a carrier-free radio transmission that uses narrow, extremely low power pulses of radio energy spread across a wide spectrum of frequencies.

The impulse pulses with the characteristics given in Table 2 can be used for the data communication within MANETs.

UWB communications are required to have a $10 \mathrm{~dB}$ fractional bandwidth of more than $20 \%$ or a $10 \mathrm{~dB}$ bandwidth of more than $500 \mathrm{MHz}$. No specific modulation is required according to FCC for UWB systems. The high spectral content of the UWB waveform gives rise to one of 
the primary advantages UWB operation for communications where a UWB system is robust against multipath fading and narrowband interference.

UWB systems offer the promise of high data rate, low susceptibility to multipath fading, high transmission security low prime power requirements, low cost and simple design.

\section{RESULTS AND DISCUSSION}

\subsection{Packet Delivery Ratio}

The packet delivery ratio is defined as the number of data packet received by destination nodes divided by number of data packets transmitted by source nodes. The
Fig. 6 depicts the improved performance of the packet delivery ratio of the nodes by using UWB compared to the conventional method. This shows the packet delivery ratio of the network has been increased considerably by $5 \%$.

\subsection{Routing Overhead Analysis}

Sending a payload of data (reliably) over a communications network requires sending more than just the desired payload data, itself. It involves sending various controls and signaling data (TCP) required for achieving the reliable transmission of the desired data. The control signaling used for communication is called overhead.

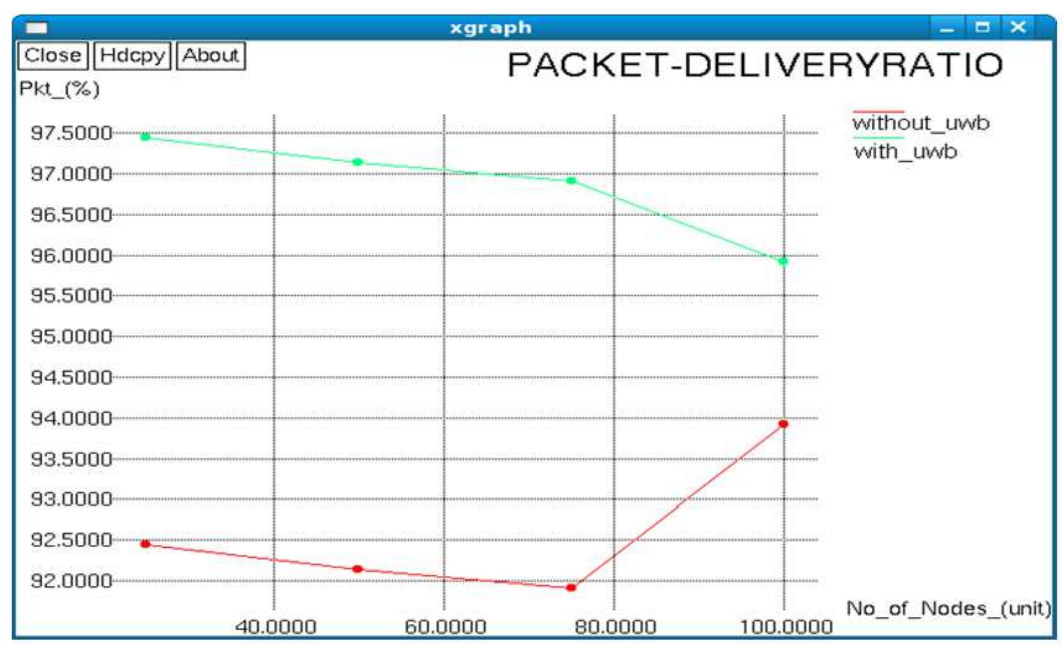

Fig. 6. Packet delivery ratio analysis with UWB and without UWB

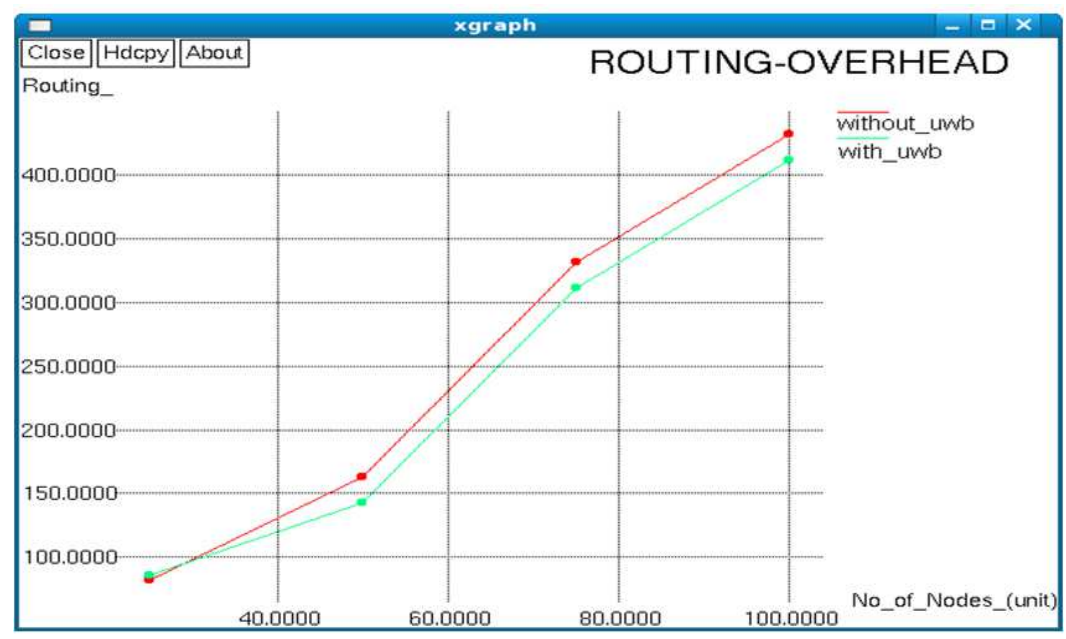

Fig. 7. Routing overhead analysis with UWB and without UWB 
The Fig. 7 depicts the improved performance of the routing overhead of the payload by using UWB compared to the conventional method. This shows the routing overhead requirement has been reduced considerably by $4.8 \%$.

The UWB based method maintains the constant packet delivery ratio which is the most desired one in real time data communications. The UWB based method uses reduced overhead which is the desired one in efficient bandwidth utilization.

\section{CONCLUSION}

For real time data communication, higher packet delivery ratio and efficient bandwidth utilization is desired by using reduced routing overhead. This can be achieved by applying impulse based radio pulses i.e., UWB method for data routing.

Simulation results show the improvement of packet delivery ratio of $5.1 \%$ and reduction in routing overhead by $4.8 \%$ for the UWB based EDNR protocol as compared with the conventional EDNR protocol.

\section{REFERENCES}

Bettstetter, C., G. Resta and P. Santi, 2003. The node distribution of the random waypoint mobility model for wireless ad hoc networks. IEEE Trans. Mobile Comput., 2: 257-269. DOI: 10.1109/TMC.2003.1233531

Gerharz, M., C. de Waal, M. Frank and P. Martini, 2002. Link stability in mobile wireless ad hoc networks. Proceedings of the 27th Annual IEEE Conference Local Computer Network, Nov. 6-8, IEEE Xplore Press, pp: 30-42. DOI: 10.1109/LCN.2002.1181761

Maleki, M., K. Dantu and M. Pedram, 2003. Lifetime prediction routing in mobile ad hoc networks. Proceedings of the WCNC 2003 Wireless Communications and Networking, Mar. 20-20, IEEE Xplore Press, New Orleans, LA, USA., pp: 1185-1190. DOI: 10.1109/WCNC.2003.1200539

Zhang, X.M., F.F. Zou, E.B. Wang and D.K. Sung, 2010. Exploring the dynamic nature of mobile nodes for predicting route lifetime in mobile ad hoc networks. IEEE Trans. Veh. Technol., 59: 1567-1572. DOI: 10.1109/TVT.2009.2038708 